\title{
Tema de Debate
}

\section{Irregularidades en la publicación de trabajos científicos}

\section{Unmethodical publication of scientific papers}

\author{
Germán Valenzuela ${ }^{1,2}$ \\ ${ }^{1}$ Cardiólogo del Hospital Rebagliati-Essalud. Lima, Perú. \\ ${ }^{2}$ Profesor Invitado de Posgrado. Universidad Nacional Mayor de San Marcos, Lima, Perú.
}

\begin{abstract}
Resumen
La publicación de un artículo científico permite al investigador transmitir sus ideas dando a conocer los resultados de su investigación, condicionando que sus hallazgos sean probados y replicados por sus colegas. Con frecuencia, la cantidad de publicaciones es usada como medición del rendimiento científico y la calidad de la misma, se evalúa también dependiendo de la revista en la cual aparece. Sin embargo, existen una serie de irregularidades en la publicación de un artículo científico, como son los aspectos relacionados a la autoría, manejo de los datos, duplicación o falsedad de las publicaciones y conflictos de interés. Palabras clave: Publicaciones de divulgación científica; autoría y coautoría en la publicación científica; mala conducta científica.
\end{abstract}

\begin{abstract}
Publication of a medical paper allows investigators to communicate their ideas and give the results of their research, conditioning they can be probed and replicated by fellow counterparts. Sometimes, the number of publications is used as a measure of scientific ranking and the quality of publications is determined by the journal in which they appear. However, we find irregularities in the publication of scientific papers, including authorship, data management, duplication or ghost publication, and conflict of interests.

Key words: Publications for science diffusion; authorship and co-authorship in scientific publications; scientific misconduct.
\end{abstract}

La publicación de un artículo científico es la meta de todo investigador debido a que contribuye a incrementar el conocimiento biomédico. Al publicar un artículo, el investigador da a conocer los resultados de su investigación, transmitiendo sus ideas y permitiendo que sus hallazgos sean probados y replicados por sus colegas. El investigador garantiza también la originalidad de su trabajo mediante la autoría. Sin embargo, al ser las publicaciones científicas fundamentales en el desarrollo científico, la cantidad de publicaciones es usada como medición del rendimiento académico de los individuos, permitiéndoles promociones en sus carreras, fondos de investigación y ayudas para el caso de los estudiantes; así como el reconocimiento individual y del medio donde se desarrolla la investigación. En los últimos años se ha comenzado a analizar la calidad de las publicaciones científicas, siendo uno de los métodos usados considerar el 'factor de impacto' de la revista en el cual el artículo ha sido publicado. Este viene a ser el número promedio de veces en las cuales el artículo de la revista en mención es citado en un período de 2 años ${ }^{(1-3)}$.

Por ello, al ser las publicaciones científicas una manera de medir la producción intelectual en el mundo académico, muchas de ellas originan conflictos entre profesionales, que afectan negativamente su motivación y sus relaciones laborales ${ }^{(2,3)}$.
A continuación, presentaré una revisión breve del tema, motivado por experiencias personales y aquellas recogidas de otros investigadores.

Caso 1.- Un médico residente realiza un trabajo científico con la autorización del jefe del servicio. Invita a los médicos del servicio a participar en el reclutamiento de los pacientes. Solo un médico asistente participa activamente en el reclutamiento, y el grupo restante entorpece sus labores porque "no cree en la utilidad de un trabajo descriptivo". Finalmente, el trabajo termina en una publicación y el autor decide colocar al médico que participó. Indignado, el jefe de servicio le comunica su malestar, debido a que por "normas internas del Servicio todos los médicos debían ser incluidos en la publicación".

Caso 2.- Un médico residente de tercer año decide participar en un concurso para médicos residentes. Organiza un grupo de investigación integrado por un médico residente de segundo año y un médico residente de primer año. Este último ha trabajado más de diez años en el Ministerio de Salud en actividades recuperativas y su edad es mucho mayor que la de los otros dos participantes. La selección de este último médico se realizó por el interés en su formación y el trato respetuoso que mantenía con su residente de rango superior. El proyecto es presentado de manera grupal, siendo ganador de un certificado y una subvención económica. El autor de la idea original asiste a la premiación y comprueba que solo el médico de mayor edad ha sido nominado. Al hacer las investigaciones respectivas escucha la versión de los organizadores de la premiación, quienes indicaron que el médico en mención, se adjudicó la plena autoría de la investigación, señalando que los dos médicos restantes eran "jóvenes que trabajaban bajo su tutela"

Caso 3.- Un médico descubre que su artículo publicado y presentado en un Congreso Nacional ha sido copiado dos años después y publicado en el libro de resúmenes del mismo congreso. El autor principal de la copia realizó su internado en el hospital donde laboró como residente; decidió copiar el trabajo e incluir a un grupo de cinco amigos como coautores, con la finalidad de obtener puntaje adicional para el concurso del residentado. En el resumen falso, los ahora médicos publicaron el trabajo como si hubiera sido realizado en un hospital diferente a donde realmente se realizó. Indignado, el autor original dirige una carta al Comité de Ética del Colegio Médico y, después de la investigación correspondiente, los autores falsos son sancionados con 4 años de imposibilidad de postular a un concurso de residentado médico a nivel nacional.

Caso 4.- Un médico con conocimientos avanzados de estadística interactúa como 
residente de tercer año con un médico residente de primer año, durante una rotación común. Al terminar sus carreras, el segundo médico comienza a obtener datos de pacientes con una enfermedad crónica "sin saber qué hacer con ellos". El médico quien había asesorado ad honorem a éste en la elaboración de su tesis de especialista, revisa una abundante bibliografía, diseña una escala nueva predictora de mortalidad y entrega los resultados, conclusiones y recomendaciones al segundo médico. Este los presenta en un congreso de su especialidad, resultando ganadores de un premio. El segundo médico, quien pudo asistir al congreso, oculta al primero el buen resultado y después de tres semanas de recibir el certificado y la subvención económica decide entregar el 30\% de lo obtenido al médico uno, expresándole que "ambos tuvieron contribuciones distintas en la investigación".

\section{AUTORÍA}

Según las raíces latinas y griegas el autor es el creador, y la autoría justifica los procesos de origen de ideas o el inicio de la ejecución de éstas. Como este concepto ha evolucionado, su proceso está relacionado ahora con la originalidad o el valor científico del trabajo publicado, con la veracidad y reproducibilidad del reporte y el reconocimiento de la propiedad del mismo ${ }^{(4)}$.

De acuerdo al comité internacional de editores de revistas médicas, cuyas siglas en inglés son ICMJE (2004), los criterios esenciales de autoría son los siguientes $(1,3,4)$ :

1. Participación con contribuciones sustanciales para la concepción y diseño, o adquisición de los datos, o el análisis e interpretación de los mismos.

2. Participación en la redacción o revisión crítica del contenido intelectual de la información obtenida.

3. Participación en la revisión final del artículo para ser publicado.

Según dicho comité, el autor debe cumplir todos los criterios arriba señalados.

La adquisición de fondos, la recolección de datos o la supervisión general del grupo de trabajo por sí mismo, no justifican la autoría. Tampoco se debe considerar autor de un trabajo al médico que permite que los pacientes ingresen al estudio o al jefe de servicio o departamento. Estos aspectos serán abordados a continuación ${ }^{(4)}$.

Según estadísticas de los Estados Unidos de Norteamérica, el número de problemas relacionados con la autoría se ha incremen- tado en la última década. Por ello, y para evitar problemas posteriores, el investigador principal deberá decidir la posición de los autores en la futura publicación, con énfasis en las participaciones más importantes, y añadiendo a los participantes restantes conforme se desarrolle la investigación ${ }^{(3,4)}$.

Algunos valores éticos relacionados con la autoría en investigación médica son ${ }^{(4,5)}$ :

1. Veracidad: cumpliendo los criterios establecidos por el método científico, revisión crítica por la revista y participando, por otro lado, en la solución de las preguntas formuladas por los lectores y en la realización de pruebas confirmatorias si fuere el caso.

2. Confiabilidad: Donde los autores no solo deberán publicar lo que consideren que es verdad, sino intentar la minimización de los sesgos de selección, rechazo o interpretación de información que se presentará a los lectores. Se negaría este valor con la formulación de juicios adelantados, tomando como base alguna información sesgada, o con el hecho de ocultar los datos no deseados por la compañía o entidad patrocinadora.

3. Reconocimiento: Relacionada con el crédito social otorgado a los creadores de un trabajo original, difícil de comprender -dependiendo del caso- cuando el número de autores que aparece en la publicación es grande.

En algunos casos, la inclusión de los autores se realiza por razones políticas o sentimentales, más que éticas; pero, los valores éticos deberán regir siempre la toma de decisiones, especialmente aquellos relacionados con el principio cardinal llamado justicia. Lo recomendado es poder señalar las funciones realizadas por cada participante, incluidas las del autor de la publicación ${ }^{(5,6)}$.

Por ello, el orden propuesto para los autores debe ser:

El 'autor principal' o 'primer autor', quien debe ser la persona que trabajó más durante el proceso de elaboración del artículo. Aún cuando no entienda todas las técnicas de recolección e información, o del análisis de los datos obtenidos, se le reconoce la capacidad de comprender el proceso por entero. Algunos ejemplos de irregularidades en la elección del primer autor serían, por ejemplo, el caso en el que un individuo participa traduciendo el artículo o realizando el análisis de datos, y no durante todo el proceso, y se adjudica el derecho de ser el primer autor, sin tener la capacidad de explicar el proceso de desarrollo de la investigación ni de responder las inquietudes de los lectores, si es que las hubieran ${ }^{(1,3,6)}$.

Los 'otros autores' participan en el grupo de investigación, en uno o más estadios del proceso de preparación del artículo; sin embargo, la posición o participación de éstos debería definirse antes de que el artículo sea publicado. Algunas irregularidades en la elección de estos autores serían ${ }^{(3,4)}$ :

- Autorías múltiples: para obtener promociones laborales, puntuaciones curriculares o premios.

- Autorías por obsequio o bajo presión, donde un individuo reclama su inclusión o la inclusión de otros miembros por su posición preferencial en un servicio o departamento.

- Autorías fantasmas, en los que la persona es removida de la publicación, habiendo cumplido con los requisitos mencionados al inicio de este artículo.

Durante el proceso de elaboración del artículo y la revisión de éste por el comité editorial de la revista, se reconoce también la 'publicación duplicada' como una irregularidad frecuente, la cual está referida a la publicación del mismo artículo en varias revistas, sin modificaciones significativas, para obtener el reconocimiento que ello condicione. Es importante que los estudiantes e investigadores jóvenes reconozcan que es una situación antiética y susceptible de ser castigada ${ }^{(3,4)}$.

Otra irregularidad es la 'publicación dividida', la cual está referida a la fragmentación de los datos para incrementar el número de publicaciones. Existen también casos como la 'publicación extendida', en la cual el artículo original es modificado no sustancialmente, añadiéndosele más datos ${ }^{(3,4)}$.

Para evitar estas irregularidades, los editores deberían estar informados de toda la documentación relevante relacionada con el artículo en revisión, como cartas al editor, copias de manuscritos (remitidos, en prensa o publicados) y cualquier artículo relacionado, los mismos que deberían ser citados en las referencias del artículo remitido ${ }^{(3,4)}$. Figura 1.

\section{MANEJO DE LOS DATOS}

Para evitar irregularidades en este rubro, debe cumplirse las reglas de buenas prácticas clínicas (GCP, en inglés). Esto significa que la industria farmacéutica estandariza los procedimientos operativos y garantiza la calidad de control de los sistemas aplicados en el desarrollo de un nuevo fármaco. Por ello, los investigadores deberán almacenar los datos obtenidos relacionados con la 
evaluación de pacientes, por un mínimo de 5 años después de finalizado el estudio, y prepararse para las evaluaciones y auditorías de la información obtenida ${ }^{(7)}$.

\section{CONFLICTOS DE INTERÉS}

El buscar la objetividad científica al conducir y llevar a cabo una investigación clínica ayuda a proteger a los lectores de artículos científicos de errores en la interpretación de los datos ${ }^{(6,8)}$.

Los conflictos podrían ser personales (no me agrada determinado individuo), comerciales (trabajo para la industria farmacéutica), políticas (estoy en contra de determinada política o legislación sanitaria o gubernamental), académicas (conozco a los autores) o financieras (he recibido pagos para escribir el artículo, o facilidades para viajes o acceso a literatura científica) ${ }^{(6,8)}$.

Los editores de revistas científicas, también cumplen un rol importante en proteger a los lectores de sesgos en la publicación de ensayos clínicos positivos. Finalmente, en el manuscrito final, estas variables deberán ser declaradas como conflictos de interés ${ }^{(6-9)}$.

\section{FRAUDE EN \\ INVESTIGACIÓN}

En el año 2002, se encontró que Jan Henrick Schön había falsificado por lo menos 17 publicaciones científicas. Este hecho fue puesto al descubierto después de que los lectores observaran un gráfico correspondiente a la estructura de una molécula que apareció en la revista Nature, y que había sido sutilmente cambiado en la revista Science, promocionando este caso como un descubrimiento distinto. Este autor, que publicaba en promedio un artículo cada 8 días, fue desacreditado después de este descubrimiento, considerándose que todas sus publicaciones no eran confiables ${ }^{(8,9)}$.

La definición de fraude en investigación está referida a una actitud deshonesta premeditada, que incluye la fabricación (invención de los datos), falsificación (distorsión de los datos) y plagio (copia de gran cantidad de datos sin la correspondiente autorización).
Según algunos autores, la ganancia económica no es siempre la motivación que condiciona un fraude, y se recomienda a los investigadores solicitar a sus compañeros de grupo la revisión individual de los datos, verificando cuidadosamente las tablas y gráficas que serán publicadas en el artículo donde aparecerán nombrados ${ }^{(7-10)}$. conocimientos. Dicho proceso deberá ser ordenado, con los más altos estándares de calidad y en un medio ambiente adecuado. Por ello, debemos rodearnos de personas que entiendan estos conceptos, sin olvidar jamás que la calidad de nuestros trabajos de investigación será el reflejo de nuestra calidad profesional, ética y moral.

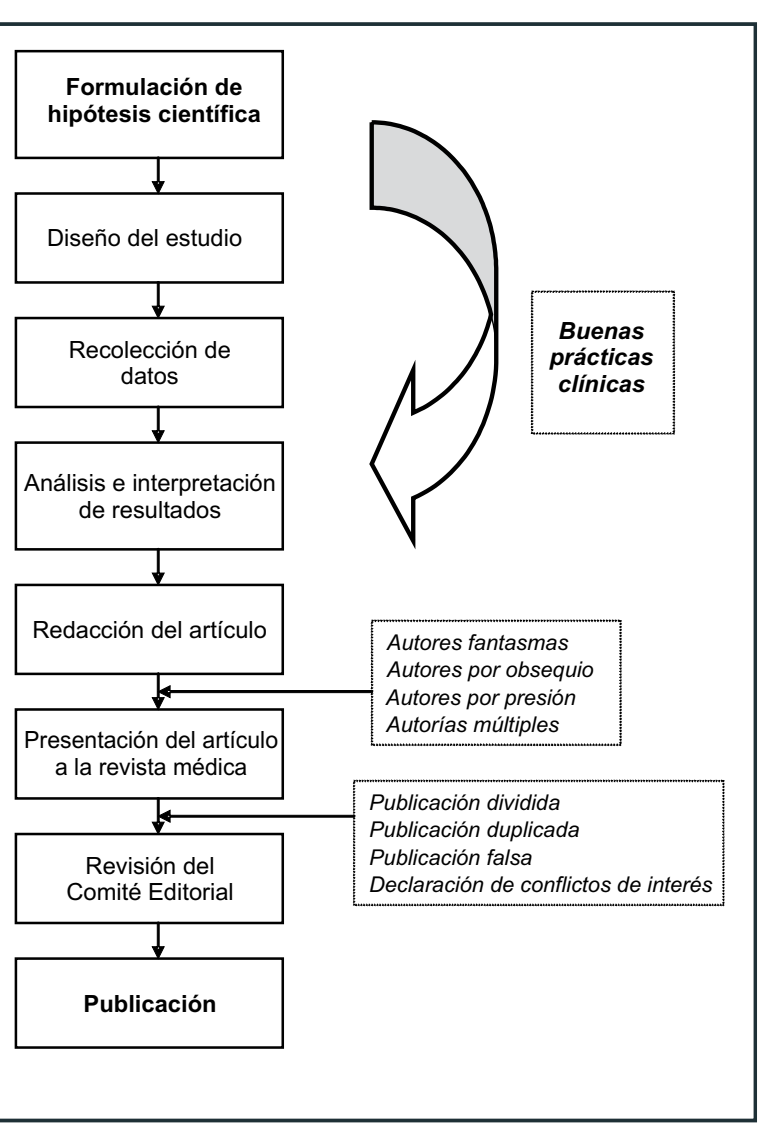

Figura 1. Irregularidades en la publicación de artículos científicos.

Debe comprenderse también que los investigadores no actúan aisladamente, y se deberá integrar las buenas prácticas clínicas para todos los miembros del grupo de investigación. Para mantener un ambiente de investigación óptimo, se recomienda la interacción con los jurados, la revisión de los proyectos y los resultados de la investigación por los jurados, así como una actitud crítica por parte de las unidades de investigación locales y durante las presentaciones en cursos y congresos. Algunos países europeos tienen comités nacionales dedicados a vigilar la veracidad de los datos clínicos obtenidos, y de aplicar las medidas correctivas cuando los procesos no se realizan de acuerdo a las reglas ${ }^{(9)}$.

Finalmente, debemos recordar que investigamos para dar solución a una pregunta, y con ello pretendemos generar nuevos

\section{REFERENCIAS BIBLIOGRÁFICAS}

1.McKneally M. Put my name on that paper. Reflections on the ethics of authorship. I Thorac Cardiovasc Surg. 2006;131:517-9.

2.Lagnado M. Increasing the trust in scientific authorship. Brit J Psych. 2003;183:3-4.

3.Carneiro MA, Cangussú SD, Fernandes GW. Ethical abuses in the authorship of scientific papers. Rev Brasileña Entomología. 2007;51(1):1-5. 4.Skeikh A. Publication ethics and the research assessment exercise: reflections on the troubled question of authorship. J Med Ethics. 2000;26:422-6.

5.Grigg J. Good research conduct. Arch Dis Child. 2005;90:229-32.

6.Hunter JM. Ethics in publishing; are we practicing to the highest possible standars? Brit J Anaesthesia. 2000;85(3):341-3.

7.Lynoe $\mathrm{N}$, Jacobsson $\mathrm{L}$, Lundgren L. Fraud, misconduct or normal science in medical research-an empirical study of demarcation. J Med Ethics.

1999;25:501-6

8. Newman A, Jones R. Authorship of research papers: ethical and professional issues for shortterm researchers. J Med Ethics. 2006; 32:4203.

9. Kwok LS. The White Bull effect: abusive coauthorship and publication parasitism. J Med Ethics. 2005;31:554-6.

10. Rennie D, Yank V, Emanuel L. When authorship fails: a proposal to make contributors accountable. JAMA. 1007;278:579-85.

Manuscrito recibido el 19 de diciembre de 2007 y aceptado para publicación el 15 de febrero de 2008.

\section{Correspondencia:}

Germán Valenzuela

Hospital Rebagliati. Servicio de Cardiología.

Av. Rebagliati s/n. Lima 11, Perú.

Correo-e: valenzuela_german@yahoo.es 